\title{
Philosophiques
}

\section{La responsabilité de la clarté}

\section{Claude Panaccio}

Volume 14, numéro 1, printemps 1987

URI : https://id.erudit.org/iderudit/027006ar

DOI : https://doi.org/10.7202/027006ar

Aller au sommaire du numéro

Éditeur(s)

Société de philosophie du Québec

ISSN

0316-2923 (imprimé)

1492-1391 (numérique)

Découvrir la revue

Citer cet article

Panaccio, C. (1987). La responsabilité de la clarté. Philosophiques, 14(1),

174-181. https://doi.org/10.7202/027006ar

Ce document est protégé par la loi sur le droit d'auteur. L'utilisation des services d'Érudit (y compris la reproduction) est assujettie à sa politique d'utilisation que vous pouvez consulter en ligne.

https://apropos.erudit.org/fr/usagers/politique-dutilisation/ 
PHILOSOPHIQUes, Vol. XIV, Numéro 1, Printemps 1987

\title{
LA RESPONSABILITÉ DE LA CLARTÉ
}

\author{
par Claude Panaccio
}

Je proposerai ici quelques réflexions que je voudrais moins originales que justes sur la question suivante: qu'est-ce qu'une société comme la nôtre est en droit d'attendre de la discipline «philosophie»? Et, bien sûr, étant moi-même philosophe de métier, il me faudra commencer par un retour sur la formulation de la question.

Par « une société comme la nôtre », je veux dire une société qui se veut démocratique, dans laquelie, donc, les décisions politiques devraient en principe émaner de ce que Jean-Jacques Rousseau appelle «la volonté générale». Je présupposerai - naïvement peut-être - que tel est bien l'idéal que nous partageons, vous et moi, celui d'une société dont chaque membre individuel se voit investi de ce que j'appellerai une «dignité politique», dont chaque membre se voit reconnu une compétence de principe à intervenir dans les débats publics, ainsi que le droit de parole nécessaire pour exercer cette compétence, une société dont chaque membre détient en politique le statut de centre décisionnel.

C'est au regard de cet idéal - présupposé - que je soulève la question de droit: qu'est-ce qu'une telle société est en droit d'attendre...? De telles questions de droit ne peuvent jamais être posées et discutées que sur le fond de certaines normes considérées - au moins provisoirement - comme admises par les interlocuteurs. Ce n'est pas là une raison de renoncer aux questions de droit. C'est tout simplement une condition sine qua non de la discussion normative, laquelle, me semble-t-il, est de toute façon inévitable. Quant à l'expression "la discipline philosophie», elle me sert à évoquer un certain nombre de pratiques institutionnalisées - je veux dire : régies par des règles dans un cadre institutionnel -, par 
exemple: l'enseignement officiellement identifié comme enseignement de philosophie - que ce soit à l'université, au collège ou à n'importe quel autre niveau -, la publication de textes identifiés comme textes philosophiques par la collection ou la revue qui les diffusent, la tenue de rencontres identifiées comme colloques ou congrès philosophiques. Enseignement, publications, colloques et congrès, voilà ce qui constitue une discipline. Le mot « philosophie » est justement le nom propre d'une telle discipline ainsi caractérisée par ses réseaux de communication et de diffusion.

Notre société consacre des ressources - que chacun d'entre nous jugera certainement insuffisantes, mais qui sont néanmoins considérables - à l'entretien de ce réseau particulier, celui de la philosophie. Il faut prendre en considération non seulement les bourses et les subventions de recherche ou de publication, mais aussi le travail professionnel d'un imposant corps professoral, l'infrastructure matérielle et organisationnelle qui appuie et rend possible le travail de ce corps professoral, le coût social de quatre cours obligatoires au niveau du collège, d'au moins dix-sept ou dixhuit programmes universitaires explicitement identifiés comme programmes de philosophie (je parle pour le Québec), dont quatre programmes de doctorat et six ou sept programmes de maîtrise. Et ainsi de suite. Décidément, la question se pose: qu'est-ce qu'une société comme la nôtre - qui se voudrait démocratique - est en droit d'attendre de la discipline philosophie?

La réponse ne peut pas être simple, parce que cette discipline qui a pour nom propre "philosophie» transporte avec elle une tradition textuelle extraordinairement riche et diversifiée dont il y a beaucoup à tirer, et de différents points de vue. Une des choses justement qu'on peut attendre de cette discipline est qu'elle diffuse et rende accessible cette longue tradition qui est la sienne de réflexion radicale et exigeante. Non pas seulement à la manière d'un conservateur de musée, mais en ravivant continuellement le dialogue avec les maîtres à penser : Platon, Aristote, ... Guillaume d'Occam et les autres.

Mais il faut aller plus loin. La philosophie se fabrique encore, elle se regénère sans cesse. Ni Thomas d'Aquin, ni Hegel, ni Marx n'en ont dit le dernier mot. Et l'on doit pouvoir attendre de la philosophie plus qu'un orgueilleux étalage de son arbre 
généalogique. On peut par exemple attendre d'elle qu'elle incarne sans relâche l'exigence de la radicalité intellectuelle, de la perpétuelle remise en question des présupposés et des préjugés, l'exigence de pousser la réflexion aussi loin qu'elle puisse aller. C'est bien là une chose qu'on est, me semble-t-il, en droit d'attendre de la discipline "philosophie » et il y en a certainement quelques autres. Je voudrais insister aujourd'hui sur l'une d'entre elles: la clarification conceptuelle.

Jean-Paul Desbiens disait récemment, à l'occasion d'une émission télévisée, que le malaise dont nous souffrons aujourd'hui n'est plus vraiment le malaise linguistique, mais bien plutôt celui de la confusion des concepts. Il n'y a qu'à suivre d'un peu plus près et d'un regard intellectueliement critique les discussions publiques sur les questions d'intérêt commun pour découvrir - avec consternation probablement - combien il a raison.

La discussion publique est un travail discursif commun par lequel, en principe, on tente, à l'aide d'arguments que l'on se sert les uns aux autres, d'arriver à des conclusions appropriées qui fassent l'objet d'un consensus entre les interlocuteurs. On aura beau se méfier tant qu'on voudra de cet idéal du consensus, y voir un instrument idéologique d'occultation et de domination et clamer à tout rompre que «dialoguer, c'est se faire avoir», on ne saurait l'abandonner purement et simplement sans laisser tomber du même coup l'idéal démocratique. Or plus une discussion est conceptuellement confuse, plus elle risque de n'aboutir à rien... ou d'aboutir à des conclusions inappropriées, ce qui est souvent encore plus grave.

On ne saurait trop insister aujourd'hui sur ce risque énorme de la démocratie, le risque de la stupidité. Le défi d'une société qui se veut démocratique est d'entretenir une discussion publique, la plus large possible, qui soit intelligente et féconde. Et cela requiert des intervenants une certaine virtuosité à manier les concepts, tout comme le système collectif de transport par véhicule individuel requiert de chaque automobiliste une certaine virtuosité à contrôler son bolide. Cela me paraît être l'un des problèmes sociaux les plus urgents à l'heure actuelle. Et la discipline philosophie trouve là une de ses grandes responsabilités sociales. Car s'il est une tâche à laquelle les philosophes se sont obstinément employés depuis 
Socrate et dont ils devraient - en principe - être devenus des experts, c'est bien celle de la clarification conceptuelle, particulièrement en ce qui a trait aux concepts les plus fondamentaux d'une conception générale du monde par exemple, ou même d'une science en particulier.

Un concept, c'est quelque chose comme le sens d'un mot. Un concept est clair pour une personne donnée si elle sait utiliser de façon appropriée le mot correspondant, si elle sait en particulier faire des inférences logiquement correctes à l'aide de ce mot ou en tout cas les reconnaître. «Ce film est pornographique » impliquet-il logiquement « il y a de la violence dans ce film»? « Respecter la vie» implique-t-il logiquement «n'enlever la vie à aucun être vivant quel qu'il soit »? Si l'on n'a pas de réponse nette à de telles questions, c'est que nos concepts de «film pornographique » ou de «respect de la vie» ne sont pas clairs pour nous.

Et la clarté est une condition sine qua non de l'argumentation coopérative par laquelle on essaie collectivement, tant bien que mal, d'en venir à un consensus sur la pornographie, l'avortement, et que sais-je encore. J'aime citer à ce sujet ce très beau passage de Karl Popper :

Voici bien des années déjà, j'avais l'habitude de mettre mes étudiants en garde contre l'idée largement répandue que l'on va à l'université pour apprendre comment parler et écrire de manière impressionnante et incompréhensible. (...) Le modèle de l'impressionnante incompréhensibilité s'oppose radicalement à ceux de la vérité et de la critique rationnelle. Car ces dernières valeurs dépendent de la clarté. On ne peut distinguer le vrai du faux, ni distinguer une réponse adéquate à un problème d'une réponse non pertinente, ni distinguer de bonnes idées d'idées banales, on ne peut évaluer des idées de manière critique, si ce n'est lorsqu'elles sont présentées avec suffisamment de clarté.

(Tiré de «Raison ou révolution? ", De Vienne à Francfort. La querelle allemande des sciences sociales, textes de Adorno, Popper, Habermas et als, Paris, Éd. Complexe, 1979, p. 242.)

Voilà donc une tâche philosophique par excellence : contribuer par l'enseignement, par la publication, par la prise de parole à la clarification des termes clés de la discussion publique - ou même de ceux de notre science, si sophistiquée et pourtant si obscure très souvent, qui, elle aussi d'ailleurs, est en principe publique - et, par là, à une meilleure identification des enjeux, 
des choix qui se présentent, des diverses positions qui s'affrontent. Socrate parcourait les rues en questionnant ses concitoyens sur la portée de leurs concepts : qu'est-ce que la justice? qu'est-ce que la beauté ? qu'est-ce que la piété ? C'est-à-dire : quel sens donnezvous au mot « justice », au mot «beauté », au mot " piété » ? C'est cette même tâche, inhérente à toute forme de démocratie, qui nous requiert encore aujourd'hui.

Et cette responsabilité, une fois assumée par la discipline - c'est-à-dire par la collectivité de personnes réelles qui incarnent à un moment donné la discipline - cette responsabilité, dis-je, justifie amplement, me semble-t-il, les ressources que notre société consacre à cette discipline.

Mais l'assumons-nous vraiment? Il faudrait en tout cas pouvoir en juger. Bien des anecdotes fort embarrassantes circulent... jusque dans les journaux. Et nous connaissons tous des collègues confus, nous connaissons tous de ces philosophes par profession qui se complaisent dans ce que Popper appelle "l'impressionnante incompréhensibilité " et qui croient, pour reprendre un mot de Schopenhauer, qu'il suffit d'être obscur pour être profond. Cela est inévitable sans doute. C'est le prix à payer pour sauvegarder le pluralisme des traditions de pensée et la possibilité de l'innovation intellectuelle, qui a souvent au premier coup d'œil des allures un peu farfelues.

Mais le spectre du dirigisme ne doit pas non plus nous faire céder à la facilité du laisser-aller. La discipline philosophie ne saurait au nom de quelque privilège insensé échapper à toute forme d'évaluation. Certes mieux vaut encore en pareil domaine la pagaille que l'orthodoxie, et j'aime mieux pour ma part des notes de cours maladroites mais personnelles qu'un cathéchisme trop bien structuré. Ce qui est requis, c'est un cheminement d'ensemble de la communauté philosophique, une maturation collective dont les instruments sont des choses comme les plans-cadres, les réunions, administratives aussi bien qu'académiques, les revues, les livres, les congrès, les colloques, les bulletins et - pourquoi pas? - les États Généraux. C'est là le rôle d'organismes comme la Société de philosophie du Québec ou la Coordination provinciale de philosophie. 
Cela est à comprendre à partir des deux principes suivants :

1) L'évaluation qualitative de ce qui se fait en philosophie, ici au Québec par exemple, ne devrait pas être évitée. Il faut admettre la notion de compétence en philosophie. Faute de quoi bien sûr, les cours que nous donnons et dans lesquels, je présume, nous prétendons apprendre quelque chose à quelqu'un, les diplômes que nous décernons, les revues que nous publions, etc., tout cela serait de la frime. Et cette nécessaire " compétence» philosophique, elle doit pouvoir être repérée. C'est le rôle de l'évaluation.

2) Cette évaluation académique doit être pour l'essentiel une évaluation par les pairs. C'est là que l'idée d'un cheminement collectif devient pertinente. Il n'y a pas d'autre voie que de s'évaluer les uns les autres, tant bien que mal et au meilleur de nos connaissances. Il y eut un temps au Québec - un temps récent - où la crainte de la répression était si grande chez les intellectuels que beaucoup d'entre eux refusaient purement et simplement toute forme d'évaluation. C'était une erreur. Dans la mesure même où les tâches dévolues à la discipline philosophie - celle par exemple de contribuer à l'entreprise toujours inachevée de clarification des concepts les plus fondamentaux - dans la mesure où ces tâches revêtent quelque importance, il est indispensable de recourir, avec tout le doigté et la prudence qui s'imposent, à certaines formes de contrôle. Ces contrôles viennent inévitablement pour une large part de l'extérieur de la discipline, c'est normal: administrations académiques, organismes gouvernementaux, groupes étudiants ont en principe leur mot à dire. Mais tout cela demeure extrinsèque et ces formes externes d'évaluation, nous le savons tous par expérience, ratent très souvent l'essentiel d'une pratique proprement philosophique. C'est pourquoi je disais: l'évaluation académique, si elle est inévitable, doit être pour l'essentiel évaluation par les pairs. Et les pairs en question auraient bien tort de se défiler au nom d'une pureté tout abstraite : qui suis-je pour juger de mes collègues? Une collectivité académique instaure habituellement pour l'évaluation par les pairs un jeu subtil de règles et de relations hiérarchisées 
dont elle ne saurait faire l'économie qu'au prix d'une renonciation suicidaire à ses responsabilités sociales. Cette hiérarchisation tient à la reconnaissance de la compétence et les formes de cette reconnaissance par la collectivité des pairs sont elles-mêmes en général reconnaissables: publications, subventions, évaluations départementales, etc. Ce système paraît souvent bien arbitraire, mais on ne saurait s'en passer entièrement. S'il ne donne pas les résultats escomptés, il n'y a pas d'autre choix que de chercher à l'améliorer. Les institutions sélectives sont souvent perçues avec beaucoup de méfiance par les intellectuels, mais ce qu'on peut leur reprocher, le cas échéant, ce n'est pas d'être sélectives, c'est d'aboutir aux mauvais choix.

Bref, une société comme la nôtre peut attendre beaucoup de la discipline philosophie, et, en particulier, une contribution décisive et exigeante à la clarification des concepts. Cette responsabilité qui incombe à notre discipline ne peut être assumée qu'au moyen - notamment - d'un système, probablement complexe, délicat et diversifié, d'auto-évaluation de la discipline par elle-même.

Département de philosophie

Université du Québec à Trois-Rivières 